\title{
BEM-ESTAR, QUALIDADE DE VIDA E ESPERANÇA EM CUIDADORES FAMILIARES DE PESSOAS COM ESQUIZOFRENIA 1
}

\author{
| Patricia Dias²; Marcos Hirata ${ }^{3}$; Fernanda Pamela Machado ${ }^{4}$; Margarita Antonia Villar Luis ${ }^{5}$; Julia Trevisan Martins ${ }^{6}$ |
}

\section{RESUMO}

INTRODUÇÃO: Com início do processo de reforma psiquiátrica no Brasil através da desinstitucionalização, foi adotado a preferência pelo tratamento psiquiátrico comunitário, inserindo o familiar no tratamento desses pacientes, gerando sobrecarga e preocupação em relação à qualidade de vida, bem-estar e esperança desses cuidadores familiares.

OBJETIVOS: Avaliar o bem-estar, qualidade de vida e esperança dos cuidadores familiares de pessoas com esquizofrenia.

MÉTODOS: Estudo descritivo transversal, realizado com 117 familiares de pessoas esquizofrênicas atendidas no Centro de Atenção Psicossocial em uma cidade do Paraná no Brasil. Foram aplicados os instrumentos World Health Organization 5-Item Well-Being (WHO-5), World Health Organization Quality of Life Abreviado (WHOQOL-Bref) e Escala de Esperança Disposicional.

RESULTADOS: Os pais foram identificados como os principais cuidadores (53,8\%), 74,4\% representados pelo sexo feminino; $35 \%$ apresentavam entre quatro e sete anos de estudo; $97,4 \%$ dos cuidadores mantinham contato diário com o familiar esquizofrênico, com média de tempo atuando como cuidador de 13,4 anos. Em relação à pontuação obtida no WHO-5, 94,9\% apresentaram abaixo de 20 pontos. Na escala WHOQOL-Bref, observou-se que o domínio de relações sociais e o domínio psicológico foram os que obtiveram menor escore, $\mathrm{m}=2,8 \mathrm{e}$ $\mathrm{m}=3,2$, respectivamente. No instrumento Escala de Esperança Disposicional, 77,8\% da amostra apresentou pontuação abaixo de 32 pontos. CONCLUSÃO: $\mathrm{O}$ estudo trouxe avanços significativos para o conhecimento em torno do cuidador familiar de pessoas com esquizofrenia $\mathrm{e}$ suas principais apreensões em relação a qualidade de vida, bem-estar e esperança, bem como o subsídio para a reformulação dos serviços e políticas de saúde mental.

\section{PALAVRAS-CHAVE: Cuidador familiar; Esquizofrenia; Qualidade de vida; Esperança}

\section{RESUMEN}

\section{"Bienestar, calidad de vida y esperanza en cuidadores familiares de personas con esquizofrenia“"}

INTRODUCCIÓN: Con el inicio del proceso de reforma psiquiátrica en Brasil a través de la desinstitucionalización, se adoptó la preferencia por el tratamiento psiquiátrico comunitario, insertando al familiar en el tratamiento de esos pacientes, generando sobrecarga y preocupación en relación a la calidad de vida, bienestar y esperanza de esos los cuidadores familiares. OBJETIVOS: Evaluar el bienestar, calidad de vida y esperanza de los cuidadores familiares de personas con esquizofrenia.

MÉTODOS: Estudio descriptivo transversal, realizado con 117 familiares de personas esquizofrénicas atendidas en el Centro de Atención Psicosocial en una ciudad de Paraná en Brasil. Se aplicaron los instrumentos de la Organización Mundial de la Salud (WHOQOL-Bref) y la Escala de Esperanza Disposicional.

RESULTADOS: Los padres fueron identificados como los principales cuidadores (53,8\%), 74,4\% representados por el sexo femenino; El 35\% presentaba entre cuatro y siete años de estudio; El 97,4\% de los cuidadores mantenían contacto diario con el familiar esquizofrénico, con promedio de tiempo actuando como cuidador de 13,4 años. En cuanto a la puntuación obtenida en el WHO-5, el 94,9\% presentó por debajo de 20 puntos. En la escala WHOQOL-Bref, se observó que el dominio de relaciones sociales y el dominio psicológico fueron los que obtuvieron menor puntuación, $\mathrm{m}=2,8$ y $\mathrm{m}=$ 3,2, respectivamente. En el instrumento Escala de Esperanza Disposicional, el $77,8 \%$ de la muestra presentó una puntuación por debajo de 32 puntos. CONCLUSIÓN: El estudio trae avances significativos para el conocimiento en torno al cuidador familiar de personas con esquizofrenia y sus principales aprehensiones en relación a la calidad de vida, bienestar y esperanza, así como el subsidio para la reformulación de los servicios y políticas de salud mental.

\section{DESCRIPTORES: Cuidador familiar; Esquizofrenia; Calidad de vida; Esperanza}

\section{ABSTRACT \\ "Well-being, quality of life and hope in family caregivers of peo- ple with schizophrenia"}

BACKGROUND: With the beginning of the process of psychiatric reform in Brazil through the deinstitutionalization, the preference was adopted for community psychiatric treatment, inserting the family member in the treatment of these patients, generating overload and a concern regarding the quality of life, well-being and hope of these patients family caregivers.

AIM: To evaluate the well-being, quality of life and hope of family caregivers of people with schizophrenia.

METHODS: A descriptive cross-sectional study was carried out with 117 relatives of schizophrenic patients attending the Psychosocial Care Center in a city of Paraná, Brazil. The World Health Organization 5-Item Well-Being (WHO-5), World Health Organization Quality of Life (WHOQOL-Bref) and Dispositional Scale of Hope instruments were applied.

RESULTS: Parents were identified as the main caregivers (53.8\%), 74.4\% were female; $35 \%$ had between four and seven years of study; $97.4 \%$ of the caregivers had daily contact with the schizophrenic relative, with an average of 13.4 years of care. Regarding the WHO- 5 score, $94.9 \%$ presented below 20 points. In the WHOQOL-Bref scale, it was observed that the domain of social relations and the psychological domain were those with the lowest score, $\mathrm{m}=2.8$ and $\mathrm{m}=3.2$, respectively. In the Dispositional Scale of Hope, $77.8 \%$ of the sample had a score below 32 points.

CONCLUSION: The study has made significant advances in the knowledge about the family caregiver of people with schizophrenia and their main apprehensions regarding quality of life, well-being and hope, as well as the subsidy for the reformulation of mental health services and policies.

\section{KEYWORDS: Family caregiver; Schizophrenia; Quality of life; Hope}

Submetido em 30-03-2019

Aceite em 10-02-2020

\footnotetext{
1 Artigo extraído de Dissertação de Mestrado "Percepção de bem-estar, qualidade de vida e esperança para cuidadores familiares de pessoas com esquizofrenia", apresentada em 2019 na Universidade Estadual de Londrina.

2 Mestre; Estudante de Pós-graduação em Enfermagem na Universidade Estadual de Londrina, Centro de Ciências da Saúde, Paraná, Brasil, patriciafrancisquini@yahoo.com.br 3 Doutor; Professor na Universidade Estadual de Londrina, Centro de Ciências da Saúde, Departamento de Enfermagem, Paraná, Brasil, mhirata@uel.br

4 Mestre; Estudante de Pós-Graduação em Enfermagem na Universidade Estadual de Londrina, Centro de Ciências da Saúde, Paraná, Brasil, fer.machado3@hotmail.com 5 Doutora; Professora Titular na Universidade de São Paulo, Escola de Enfermagem de Ribeirão Preto, São Paulo, Brasil, margarit@eerp.usp.br 6 Doutora; Professora na Universidade Estadual de Londrina, Centro de Ciências da Saúde, Departamento de Enfermagem, Paraná, Brasil, jtmartins@uel.br

Citação: Dias, P., Hirata, M., Machado, F. P., Luis, M. A., \& Martins, J. T. (2020). Bem-estar, qualidade de vida e esperança em cuidadores familiares de pessoas com esquizofrenia. Revista Portuguesa de Enfermagem de Saúde Mental (23), 23-30.
} 


\section{INTRODUÇÃO}

No Brasil, a partir de 1980 ocorreu o início do processo de reforma psiquiátrica e a reabilitação psicossocial por meio da desinstitucionalização. O Estado, então, passou a adotar como política a preferência pelo tratamento psiquiátrico comunitário, visando ampliar, assim, a participação familiar no tratamento desses pacientes, bem como a reinserção social (Tabeleão, Tomasi e Quevedo, 2014). Essa mudança de cenário ocasionou uma melhoria em relação ao tratamento humanizado, priorizando a reabilitação psicossocial do paciente no seu meio. Entretanto, assumir o papel de cuidar e ressocializar essa pessoa, somado ao despreparo para exercer tal função, acabou por gerar no familiar sobrecarga, tornando-se assim um desafio (Oliveira, Eloia, Lima, Eloia, e Linhares, 2017; Reis, Dahl, Barbosa, Teixeira e Delgado, 2016).

Maurin e Boyd (1990), definiram a sobrecarga como o impacto causado no meio familiar pela tarefa de cuidar do paciente com transtorno mental, envolvendo aspectos físicos, emocionais, econômicos e sociais, ocasionando alterações nas atividades diárias. A sobrecarga objetiva pode ser caracterizada pelo excesso de assistência ao paciente, por meio de gastos financeiros, mudanças na rotina social, profissional ou pessoal, adequação do ambiente em que se vive, ou seja, as atividades cotidianas que precisam ser realizadas para suprir a necessidade do indivíduo doente. Já a sobrecarga subjetiva é assimilada ao grau de incômodo percebido ou avaliado pelo cuidador familiar, suas reações emocionais, sentimentos de sobrecarga pela responsabilidade de cuidar, ligados, então, a fatos referentes às consequências negativas da sobrecarga do ato de cuidar.

As doenças mentais são consideradas doenças crônicas, que causam fragilidade e incapacidade para o portador, e são cercadas de estigmas e preconceitos de toda a sociedade (Perkins et al., 2018). Nesse contexto das doenças mentais, a esquizofrenia é considerada a doença mais incapacitante quando nos referimos a transtornos mentais, e é definida pela Classificação Internacional de Doenças (CID-10) da Organização Mundial da Saúde (OMS) (2007) como: "Distorções fundamentais e características do pensamento e da percepção, e por afetos inapropriados ou embotados. Envolvem fenômenos psicopatológicos como transtornos de pensamentos, ideias delirantes e vozes alucinatórias." (p.317), por esse motivo o cuidado tornou-se ainda mais árduo, principalmente para o familiar responsável pelo cuidado.
O Ministério da Saúde na Portaria n. ${ }^{\circ} 3.088$, de 23 de dezembro de 2011, institui a Rede de Atenção Psicossocial (RAPS), cuja finalidade é a criação, ampliação e articulação de pontos de atenção à saúde para pessoas com sofrimento ou transtorno mental e com necessidades decorrentes do uso de crack, álcool e outras drogas. No âmbito do Sistema Único de Saúde (SUS), definiu e estabeleceu diretrizes para o funcionamento dos serviços em todos os níveis assistenciais, no qual a assistência prestada ao paciente devia incluir o atendimento à família e o desenvolvimento de atividades comunitárias, com foco na integração do paciente na comunidade e inserção familiar e social.

Nesse contexto, verificou-se que há necessidade de que os serviços de saúde mental utilizem ações, técnicas ou métodos que propiciem melhores resultados de tratamento, particularmente em relação aos familiares, devido ao seu alto grau de estresse e sobrecarga, podendo assim, interferir no bem-estar, qualidade de vida e esperança desses cuidadores familiares.

Um conceito muito difundido de bem-estar está organizado em duas perspectivas, sendo uma o bem-estar subjetivo (BES), ligado a uma visão de bem-estar como prazer, felicidade e satisfação com a vida, ou seja, "sentir a vida" e "pensar sobre ela", já o bem-estar psicológico (BEP), consiste no desenvolvimento humano e na capacidade de superar dificuldades (Ryff, 1989).

O termo qualidade de vida (QV) aparece sempre com sentido bastante genérico, porém vem sendo muito explorado e possui diversas definições, dependendo da teoria que o originou. A OMS, por meio de um grupo de estudiosos em QV, o World Health Organization Quality of Life (WHOQOL), definiu QV como “[...] a percepção do indivíduo de sua posição na vida no contexto da cultura e sistemas de valores nos quais ele vive e em relação aos seus objetivos, expectativas, padrões e preocupações [...]" (World Health Organization [WHO], 1998, p.3).

Já a Esperança é um estado emocional positivo, com percepções voltadas para a obtenção de um objetivo, composto pela interação entre rotas e agenciamentos, sendo as rotas o caminho planejado para obtenção desse objetivo, e o agenciamento a motivação para obter tal objetivo (Snyder et al., 1991).

Há diversos estudos relacionados à sobrecarga objetiva e subjetiva e com a utilização de escalas em diversos tipos de cuidadores, porém cria-se uma lacuna referente ao cuidador familiar de pessoas com esquizofrenia com a utilização de escalas para mensuração do bem-estar, qualidade de vida e esperança destes cuidadores. 
Opoku-Boateng et al (2017) expuseram que cuidadores de pessoas com esquizofrenia apresentavam baixa qualidade de vida, interferindo desta forma, negativamente em suas rotinas.

Desta forma, por tratar-se de uma tarefa com impactos importantes no ambiente familiar, infere-se que possa existir uma gama de outros construtos subjetivos ainda pouco estudados, que associados a sobrecarga e estresse, podem complementar a avaliação da amplitude do enfrentamento dessa problemática do cuidador familiar. Sendo assim, faz-se necessário avaliar o bem-estar, qualidade de vida e esperança dos cuidadores familiares de pessoas com esquizofrenia.

\section{MÉTODOS}

Tratou-se de um estudo do tipo descritivo, transversal e correlacional. Fizeram parte da pesquisa, cuidadores familiares de pacientes esquizofrênicos adultos atendidos no Centro de Atenção Psicossocial III (CAPS III) em uma cidade de grande porte no norte do estado do Paraná, no Brasil.

Foram selecionados todos os pacientes com diagnóstico de esquizofrenia, de acordo com o Código Internacional de Doenças (CID-10) compreendido pelos códigos de F-20.0 a F-20.9, que constavam nos registros do serviço. Com isso, a população de pacientes e seus respectivos familiares foi quantificada em 320 casos. Para o cálculo estatístico, foi utilizado o programa IBM Sample Power v.3.0, poder de análise de $80 \%$, perdas de $10 \%$ e erro padrão médio de 0,2 . Com isso, a partir desses parâmetros, a amostra necessária foi estimada em 114 familiares.

Foi realizado o levantamento de dados por meio das fichas elaboradas pelos profissionais (psicólogos, enfermeiros, assistentes sociais) atuantes no CAPS III, no qual cada um desses profissionais é responsável por uma área do município (Região Norte, Sul, Leste, Oeste e Centro), totalizando 320 casos de pacientes esquizofrênicos em acompanhamento. Foram separados os respectivos prontuários, para que, de forma aleatória, fossem escolhidos os casos a serem pesquisados. Houve um processo de amostragem aleatória, na qual foi elaborada uma sequência numérica para randomização e posterior sorteio de 175 prontuários. Após levantamento de dados desses prontuários, foram revisadas as agendas de consultas de cada região, a fim de conciliar as datas de consulta dos pacientes sorteados para realização da entrevista.
Para os familiares que não foram encontrados nesse primeiro momento, foram realizados até três contatos telefônicos, visando informar o motivo da ligação e da entrevista. A ausência do familiar na data e hora previamente agendadas foi considerada como perda. Dos 175 casos listados, obtivemos uma exclusão (não demonstrava entendimento frente às questões), 17 recusas em participar da pesquisa e 40 perdas, totalizando 117 entrevistas.

As entrevistas foram realizadas pela própria pesquisadora com os familiares durante o período de permanência no CAPS III em virtude da consulta e do grupo de familiares, sendo utilizados quatro instrumentos, e tempo de aplicação de aproximadamente 20 minutos. Os dados foram digitados foram digitados diretamente na plataforma online Google forms, e após, tabulados no programa Statistical Package for The Social Sciences (SPSS). As entrevistas ocorreram entre os meses de dezembro de 2017 e junho de 2018.

Para a coleta de dados, foram utilizados quatro instrumentos. Primeiro, um questionário para caracterização sociodemográfica e econômica. Para avaliação do bemestar, foi utilizado o questionário World Health Organization 5-Item Well-Being Index (WHO-5) ou Índice de Bem-Estar da Organização Mundial da Saúde. O questionário é composto de cinco questões com valores de 0 a 5 pontos em uma escala Likert, tendo o escore total variando de 0 a 25 , e visa a medir o bem-estar mental atual. Possui versão validada para o Brasil por Souza e Hidalgo (2012), com o alfa de Cronbach =0,83. A mesma autora indica que uma pontuação menor que 20 pode sugerir presença de transtorno depressivo, e, segundo Bech (2004), escores menores ou iguais a 13 indicam comprometimento do bem-estar e sugerem análises complementares para depressão.

Para avaliação da QV, foi utilizado o World Health Organization Quality of Life Instrument (WHOQOLBref) ou Instrumento de Avaliação de Qualidade de Vida Abreviado da Organização Mundial da Saúde, é composto de 26 questões, duas delas relacionadas à qualidade de vida geral - Índice Geral de Qualidade de Vida (IGQV) -, e as outras 24 questões compõem quatro domínios: físico, psicológico, relações sociais e meio ambiente. Trata-se de um instrumento autoaplicável, rápido e de fácil compreensão. As respostas seguem de acordo com uma escala Likert (variando de 1 - muito ruim; muito insatisfeito; nada; nunca, a 5 - muito boa; muito satisfeito; extremamente; sempre); conforme maior a pontuação, melhor a QV do indivíduo. 
O WHOQOL-Bref foi traduzido e validado no Brasil por Fleck et al (2000) e apresentou boa consistência interna, com o coeficiente de Cronbach variando entre 0,71 e 0,84 entre os domínios.

E para avaliação da esperança, foi utilizada a Adult Dispositional Hope Scale, ou Escala de Esperança Disposicional, instrumento adaptado e validado no Brasil por Pacico, Bastianello e Hutz (2013) com coeficiente de confiabilidade alfa de Cronbach de 0,79 . Esse instrumento conta com 12 itens distribuídos igualmente entre rotas, agenciamentos e itens filtro, de acordo com uma escala Likert de cinco pontos (sendo $1=$ totalmente falsa, e $5=$ totalmente verdadeira) para avaliar o nível de concordância com cada uma das questões.

O cálculo do escore é feito somando a pontuação obtida nas questões $1,4,6,8$ (rotas) e 2, 9, 10, 12 (agenciamentos); as questões 3, 5, 7 e 11 não são utilizadas, pois são consideradas apenas itens distratores. Para a interpretação dos resultados, é utilizada uma tabela de acordo com os percentis e escores brutos conforme a faixa etária.

Para as análises estatísticas, foi utilizado o Statistical Package for the Social Sciences (SPSS) versão 25.0. Foram realizadas análises descritivas utilizando frequência, porcentagem, média, desvio padrão (DP), e para avaliar a correlação entre idade e as variáveis do WHOQOL-Bref foi utilizado o coeficiente de correlação de Spearman, visto que os dados não possuíam distribuição normal, sendo necessário um teste não paramétrico; e análises de consistência interna das escalas por meio do Coeficiente Alfa de Cronbach, apresentando Alfa de Cronbach de 0,74 para o Índice de Bem-Estar (WHO-5), 0,86 para o WHOQOL-Bref e 0,75 para a Escala de Esperança Disposicional.

Esta pesquisa foi aprovada pelo Comitê de Ética em Pesquisa com Seres Humanos, e contemplou a assinatura do Termo de Consentimento Livre e Esclarecido.

\section{RESULTADOS}

Foram entrevistados 117 cuidadores familiares de pessoas com diagnóstico de esquizofrenia acompanhados no CAPS III em uma cidade de grande porte do norte do estado do Paraná - Brasil.

Os pais foram identificados como os principais cuidadores $(53,8 \%)$. Do total de cuidadores, $74,4 \%$ era do sexo feminino, casada $(73,5 \%)$ e com média de idade de 56,7 anos ( $\mathrm{DP} \pm 15,7$ anos), com idade mínima de 18 anos e máxima de 83 .
Quanto à escolaridade, 35\% dos cuidadores apresentavam entre quatro e sete anos de estudo. A renda familiar predominante estava entre um e dois salários mínimos $(53,8 \%)$, sendo essa atribuída principalmente ao benefício recebido pelo paciente em virtude de sua doença. Do total, 97,4\% dos cuidadores mantinha contato diário com o familiar esquizofrênico, e a média de tempo atuando como cuidador foi 13,4 anos (DP $\pm 8,7$ anos), com variação entre 2 e 40 anos.

Com o objetivo de avaliar o nível de bem-estar da população estudada, foi utilizado o instrumento Índice de Bem-Estar (WHO-5). A média das respostas variou entre 2,23 e 2,85 , sendo considerada uma baixa pontuação.

Em relação à pontuação obtida, apenas 5,1\% dos cuidadores apresentaram pontuação maior que 20; $94,9 \%$ apresentaram abaixo de 20 pontos. Entre esses 111 cuidadores $(94,9 \%)$ que obtiveram abaixo de 20 pontos, 64 $(54,7 \%)$ apresentaram pontuação igual ou menor que 13, conforme observa-se na Tabela 1.

Tabela 1 - Pontuação obtida no Índice de Bem-Estar (WHO-5) pelos cuidadores familiares

\begin{tabular}{|l|c|c|}
\hline Pontuação & $\mathrm{n}$ & $\%$ \\
\hline 20 pontos ou mais & 6 & 5,1 \\
\hline Abaixo de 20 pontos & 111 & 94,9 \\
\hline Abaixo de 13 pontos & 64 & 54,7 \\
\hline
\end{tabular}

Quando realizada a média dos escores, que se obteve uma média geral de 13,56, e, ao analisar separadamente por gênero, 14,33 foi a média obtida para homens, e 12,79 para mulheres.

Tabela 2 - Medidas de tendência central descritiva dos domínios de qualidade de vida do instrumento WHOQOL-Bref

\begin{tabular}{|l|l|l|l|l|l|}
\hline Domínios & $\mathrm{n}$ & Mínimo & Máximo & Média & $\mathrm{DP}$ \\
\hline $\begin{array}{l}\text { Qualidade de vida } \\
\text { geral }\end{array}$ & 117 & 1,50 & 4,50 & 3,37 & 0,53 \\
\hline $\begin{array}{l}\text { Qualidade de vida } \\
\text { - Domínio Físico }\end{array}$ & 117 & 2,14 & 4,71 & 3,54 & 0,56 \\
\hline $\begin{array}{l}\text { Qualidade de } \\
\text { vida - Domínio } \\
\text { Psicológico }\end{array}$ & 117 & 2,17 & 4,17 & 3,23 & 0,43 \\
\hline $\begin{array}{l}\text { Qualidade de } \\
\text { vida - Domínio } \\
\text { Relações Sociais }\end{array}$ & 117 & 1,00 & 4,00 & 2,76 & 0,65 \\
\hline $\begin{array}{l}\text { Qualidade de vida } \\
\text { - Domínio Meio } \\
\text { Ambiente }\end{array}$ & 117 & 2,25 & 4,00 & 3,32 & 0,39 \\
\hline
\end{tabular}


Para avaliar a QV, foi utilizada a escala WHOQOL-Bref. Ao analisar as médias, o domínio de relações sociais e o domínio psicológico foram os que obtiveram menor escore, $\mathrm{m}=2,8$ e $\mathrm{m}=3,2$ respectivamente, seguidos pelo domínio meio ambiente $(\mathrm{m}=3,3)$ e pelo domínio físico $(\mathrm{m}=3,5)$, como é possível observar na Tabela 2 .

Os dados da Tabela 3 mostram as correlações entre a idade e os domínios do WHOQOL-Bref. Ao analisar as correlações entre a idade e os domínios do WHOQOL-Bref, ambos os domínios se apresentam correlacionados negativamente, com associação mais forte especialmente no domínio de relações sociais $(\mathrm{r}=-0,401 /$ $\mathrm{p}<0,01)$.

Tabela 3 - Coeficiente de Correlação de Spearman entre a idade e os domínios de qualidade de vida do instrumento WHOQOL-

Bref do cuidador familiar de pessoas com esquizofrenia

\begin{tabular}{|l|c|c|c|c|c|}
\hline & IGQV(1) & $\begin{array}{c}\text { Domínio } \\
\text { Físico }\end{array}$ & $\begin{array}{c}\text { Domínio } \\
\text { Psicológico }\end{array}$ & $\begin{array}{c}\text { Domínio } \\
\text { Meio Am- } \\
\text { biente }\end{array}$ & $\begin{array}{c}\text { Domínio } \\
\text { Relações } \\
\text { Sociais }\end{array}$ \\
\hline $\mathrm{r}$ & $-0,193^{\star}$ & $-0,310^{* *}$ & $-0,142$ & $-0,221^{\star}$ & $-0,401^{* *}$ \\
\hline P valor & 0,037 & 0,001 & 0,127 & 0,016 & 0,000 \\
\hline
\end{tabular}

*correlação estatisticamente significativa $(\mathrm{p}<0,05) ;{ }^{* *}$ correlação estatisticamente significativa $(\mathrm{p}<0,01)$.

(1)IGQV = Índice Geral de Qualidade de Vida

Para avaliação da esperança, foi utilizada a Escala de Esperança Disposicional. O escore bruto considerado normal para população adulta é de 32 (média de 31,6). Conforme apresentado na Tabela 4, apenas 4,3\% da amostra estudada apresentou o escore aceitável, e $17,9 \%$ apresentaram acima de 32 pontos, 77,8\% apresentou escores abaixo de 32 , com média de 27,9, sendo considerado baixo nível de esperança.

Tabela 4 - Frequência e porcentagem obtidas pelos cuidadores familiares na Escala de Esperança Disposicional

\begin{tabular}{|l|c|c|}
\hline Pontuação & $\mathrm{n}$ & $\%$ \\
\hline Acima de 32 pontos & 21 & 17,9 \\
\hline 32 pontos & 5 & 4,3 \\
\hline Abaixo 32 pontos & 91 & 77,8 \\
\hline
\end{tabular}

\section{DISCUSSÃO}

Estudos brasileiros realizados nos estados do Rio de Janeiro (Reis et al., 2016) e Ceará (Oliveira et al., 2017), identificaram as mães como sendo as principais cuidadoras desses indivíduos.
Os resultados do presente estudo corroboram essa tendência, pois, na amostra estudada, houve prevalência de mulheres, casadas e com média de idade de 56,7 anos, sendo principalmente representadas pelas mães dos indivíduos com esquizofrenia.

Estudos mostraram que tal resultado deve-se pela distribuição dos cuidados pelos membros da família, sendo culturalmente o cuidado um padrão típico de obrigação conforme o grau de parentesco, tornando, assim, pais e cônjuges os mais envolvidos em tal tarefa (Nolasco, Bandeira, Oliveira, e Vidal, 2014).

Os cuidadores familiares mantinham contato diário com o familiar esquizofrênico $(97,4 \%)$ e atuavam por um período longo como cuidador ( $\mathrm{m}=13,46$ anos). Esses fatos, segundo estudo de Tabeleão et al (2014) podem ser atribuídos à não quebra de um ciclo de dependência, na qual normalmente espera-se que, com o passar dos anos, essas pessoas se tornem adultas e mais independentes, o que na maioria das vezes, não ocorre com os portadores de doenças psíquicas, fazendo com que esse ciclo de independência não seja característico desse grupo, mantendo, assim, a responsabilidade em cuidar integralmente e por um longo período de tempo voltada aos pais, ou parentes mais próximos responsáveis pela criação.

Em relação à escolaridade e à renda familiar, estudos nacionais realizados com cuidadores familiares de pessoas com transtorno mental (Oliveira et al., 2017; Reis et al., 2016), mostraram resultados semelhantes, nos levando a inferir, que a carga de trabalho impede que o cuidador mantenha um vínculo empregatício, ou até mesmo, apresente disponibilidade para se desenvolver academicamente. Devemos refletir quanto a esses fatores, pois o nível de escolaridade pode influir nos sentimentos dos cuidadores, e a pouca aprendizagem pode dificultar a compreensão do diagnóstico e tratamento do paciente e a baixa renda familiar, somada à responsabilidade e sobrecarga do cuidar, podem implicar negativamente.

Ao analisarmos os resultados obtidos com a WHO-5, 94,9\% apresentaram abaixo de 20 pontos, o que, segundo o estudo validado no Brasil (Souza e Hidalgo, 2012), sugere presença de transtorno depressivo. Entre esses 111 cuidadores $(94,9 \%)$ que obtiveram abaixo de 20 pontos, $64(54,7 \%)$ apresentaram pontuação igual ou menor a 13 , indicando comprometimento do bemestar, sendo necessário análises complementares para avaliação de depressão, conforme sugere Bech (2004). 
Ao avaliarmos a média dos escores da WHO-5, obtemos uma média geral de 13,56 , sendo 14,33 para homens e 12,79 para mulheres. Em estudo nacional realizado no Sul do Brasil com indivíduos da área rural, encontramos médias similares em relação ao gênero, porém com maiores escores, sendo 19,15 para homens e 17,87 para mulheres (Carvalho, Souza, e Hidalgo, 2018), o mesmo ocorre em outro estudo internacional (LucasCarrasco, 2012) realizado na Espanha com idosos, no qual encontramos média de 15,7 , sendo 16,4 para homens e 15,0 para mulheres, concluindo que menores escores de bem-estar psicológico se correlacionam com o sexo feminino, nos levando a inferir que tal fato pode estar relacionado ao acumulo de funções desempenhados pela mulher, que além de cuidadora, é também dona de casa, mãe e esposa, elevando o nível de sobrecarga. Esse evento torna-se um fator preocupante, visto que se trata de mais da metade da amostra estudada, podendo ser uma condição de alerta para os serviços de saúde sobre a necessidade de um suporte especial para as cuidadoras, uma vez que as mesmas são peças fundamentais no cuidado, tratamento e reabilitação social do paciente.

Com o instrumento WHOQOL-Bref, observou-se que os domínios que obtiveram menores escores foram o psicológico $(\mathrm{m}=3,2 / \mathrm{DP}=0,43)$ e o de relações sociais $(m=2,8 / D P=0,65)$. Tal evento deve-se ao fato de as questões norteadoras do domínio psicológico serem voltadas a retratar o sentido da vida, modo de aproveitar a vida, concentração, autoestima e aceitação da aparência física, satisfação; e o domínio de relações sociais, retratar as relações pessoais com os amigos, familiares e a vida sexual, questões que são veementemente prejudicadas ou mesmo anuladas com a intensa exposição à jornada de cuidado, pressão e responsabilidade em torno desses cuidadores.

Foi encontrado apenas um estudo realizado na República de Gana, na África com cuidadores familiares de pacientes esquizofrênicos em torno da avaliação da QV com utilização da escala WHOQOL-Bref. Os autores expõem que a população estudada apresentou baixa $\mathrm{QV}$, principalmente no domínio físico, seguido pelo domínio psicológico. Tal fato, segundo os autores, está relacionado ao programa de intervenção social a saúde do país não cobrir serviços para a esquizofrenia ou outros transtornos mentais, desta forma, sobrecarregando ainda mais os familiares (Opoku-Boateng et al., 2017).
Quando observamos que a nossa amostra apresenta resultados diferentes a esses estudos, podemos concluir que, mesmo sendo ambos cuidadores de pessoas com esquizofrenia, diferem-se nas características dos países, sistemas de saúde e apoio social.

No estudo nacional realizado com pacientes com doença óssea crônica, não foi encontrada correlação entre idade e os domínios do WHOQOL-Bref (Castro et al., 2017), já no presente estudo, houve correlação negativamente significativa entre idade e o domínio de relações sociais $(r=-0,401)$, podendo-se inferir que no cuidador familiar de pessoas esquizofrênicas, conforme maior a idade, pior é o desenvolvimento das relações sociais.

Em relação à escala de Esperança Disposicional, quase $78 \%$ da população estudada apresentou escores abaixo de 32, com média de 27,9. Visto que a esperança passar a existir quando o indivíduo tem como objetivo uma meta que deseja alcançar, dispondo de caminhos e motivação para chegar até ela, é possível que o fato de ter que cuidar de um doente crônico, frágil e sujeito a incapacidades possa interferir na autoconfiança, fazendo perder, assim, sua motivação e, consequentemente, diminuindo os níveis de esperança.

Em estudo australiano, realizado com idosos foram obtidos médias de esperança ainda menores se comparadas ao presente estudo, com média de 25,5 , podendo esse dado ser atribuído ao comprometimento funcional ocasionado pelo envelhecer. Ainda, segundo a autora a esperança atua como fator de proteção para o desenvolvimento de sintomas depressivos (Trezise, Mclaren, Gomez, Bice, \& Hodgetts, 2017).

Neste contexto, estudos nacionais sugerem a importância do acolhimento familiar em torno das dificuldades vivenciadas, afim de minimizar o grau de sobrecarga gerada pelo ato de cuidar diariamente, e ainda recomenda, o cuidador familiar do paciente esquizofrênico deve ser visto não somente como uma pessoa que cuida de seu ente, mas sim, como um paciente, devendo ser incluído em um programa intenso de tratamento e acompanhamento médico de saúde mental (Reis et al., 2016; Tabeleão et al., 2014).

\section{CONCLUSÕES}

Os resultados evidenciaram que o familiar se tornou fundamental fonte de cuidado, ligado ao processo de reconstrução da cidadania da pessoa com esquizofrenia, sendo representado principalmente pelas mães. 
A intensa jornada de cuidados sem conhecimento e sem preparo é condição de risco vivenciada por esses cuidadores familiares, sendo verificado resultados sugestivos de presença de transtorno depressivo, conforme outros estudos anteriormente realizados. Fatores como a falta de suporte social e sentimentos negativos foram pontos que favoreceram negativamente a $\mathrm{QV}$ nos domínios psicológico e de relações sociais. Houve também a presença de baixos níveis de esperança se comparado à população geral.

Entre as limitações deste estudo, a principal pode ser considerada a falta de estudos relacionados ao cuidador familiar de pessoas com esquizofrenia, e especialmente quanto à utilização de escalas para mensurar os construtos subjetivos sentidos e vivenciados por essa população.

\section{IMPLICAÇÕES PARA A PRÁTICA CLÍNICA}

Este estudo trouxe avanços significativos para o conhecimento sobre os construtos subjetivos em torno do cuidador familiar da pessoa com esquizofrenia e suas principais apreensões em relação a sua $\mathrm{QV}$, bem-estar e esperança, pretendendo alertar para o sofrimento psicológico vivenciado por esses cuidadores.

$\mathrm{O}$ fato de que $\mathrm{o}$ ato de cuidar de pessoas com esquizofrenia implica em desgaste físico e psicológico, e de ter como consequência a diminuição da qualidade de vida, do sentimento de bem-estar, acompanhado da probabilidade de desenvolvimento de transtorno depressivo e consequente diminuição da proatividade desses cuidadores familiares, deve servir para alertar os gestores das instituições quanto aos efeitos negativos que essa situação pode vir a ocasionar, e desta forma subsidiar a reformulação dos serviços e políticas de saúde mental, por meio do desenvolvimento de estratégias nos grupos de famílias, com esclarecimento de dúvidas, inclusão desses cuidadores familiares em um tratamento e acompanhamento médico, a fim de tentar prevenir sofrimento psíquico, auxiliando na melhoria do bemestar, da QV e da esperança desses cuidadores familiares e consequentemente, uma melhoria na condução do tratamento do seu familiar com esquizofrenia.

\section{REFERÊNCIAS BIBLIOGRÁFICAS}

Bech, P. (2004). Measuring the dimension of psychological gerenal well-being by the WHO-5. Quality of Life Newsletter, n.32, p.15-16.
Portaria GM/MS n. 3.088 (2011, 23 dezembro). Institui a Rede de Atenção Psicossocial para pessoas com sofrimento ou transtorno mental e com necessidades decorrentes do uso de crack, álcool e outras drogas, no âmbito do Sistema Único de Saúde (SUS). Diário Oficial [da] União. Brasília (DF). Disponível em: https://bvsms.saude.gov.br/bvs/saudelegis/gm/2011/ prt3088_23_12_2011_rep.html

Carvalho, F. G.; Souza, C. M. De; Hidalgo, M. P. L. (2018). Work routines moderate the association between eveningness and poor psychological well-being. PLoS One, 13(4), e0195078. doi: 10.1371/journal.pone.0195078

Castro, G. R. W. de, Castro, S. A. F. de, Pereira, I. A., Zimmermann, A. F., Toscano, M. A., Neves, F. S., Scottinni, M. A., Paupitz, J., Rosa, J. S. da, Buss, Z., Frode, T. S. (2017). Determinants of quality of life in Paget's disease of bone. Revista Brasileira de Reumatologia, 57(6), 566-573. doi: 10.1016/j.rbre.2017.06.002

Fleck M.P.A.; Louzada S.; Xavier M.; Chachamovich E.; Vieira G.; Santos L.; Pinzon V. (2000). Aplicação da versão em português do instrumento abreviado de avaliação da qualidade de vida "WHOQOL-bref". Revista de Saúde Pública, 34(2): 178-183. doi: 10.1590/ S0034-89102000000200012

Lucas-Carrasco, R. (2012). Reliability and validity of the Spanish version of the World Health OrganizationFive Well-Being Index in elderly. Psychiatry and Clinical Neurosciences, 66(6), 508-513. doi: 10.1111/j.14401819.2012.02387.x

Maurin, J.T.; \& Boyd, C.B. (1990). Burden of mental illness on the family: a critical review. Archives of Psychiatric Nursing, 4(2): 99-107. doi: 10.1016/08839417(90)90016-E

Nolasco, M.; Bandeira, M.; Oliveira, M. S. De; Vidal, C. E. L. (2014). Sobrecarga de familiares cuidadores em relação ao diagnóstico de pacientes psiquiátricos. Jornal Brasileiro de Psiquiatria, 63(2), 89-97. doi: 10.1590/0047-2085000000011

Oliveira, E. N.; Eloia, S. M. C.; Lima, D. Dos S.; Eloia, S. C.; Linhares, A. M. F. (2017). Family needs a break: it takes care of people with mental disorder. Revista Fund Care Online, 9(1), 71-78. doi: 10.9789/2175-5361.2017. v9i1.71-78 
Opoku-Boateng, Y. N.; Kretchy, I. A.; Aryeetey, G. C.; Dwomoh, D.; Decker, S.; Agyemang, S. A.; \& Nonvignon, J. (2017). Economic cost and quality of life of family caregivers of schizophrenic patients attending psychiatric hospitals in Ghana. BMC Health Services Research, 17(S2). doi: 10.1186/s12913-017-2642-0

Organização Mundial da Saúde. (2007). Classificação Estatística Internacional de Doenças e Problemas Relacionados à Saúde (CID-10). (10a ed.). São Paulo: Editora da Universidade de São Paulo.

Pacico, J. C.; Bastianello, M. R.; Hutz, C. S. (2013). Adaptation and validation of the dispositional hope scale for adolescents. Psicologia Reflexão e Crítica., 26(3): 488-492. doi: 10.1590/S0102-79722013000300008

Perkins, A.; Ridler, J.; Browes, D.; Peryer, G.; Notley, C.; \& Hackmann, C. (2018). Experiencing mental health diagnosis: a systematic review of service user, clinician, and carer perspectives across clinical settings. The Lancet Psychiatry, 5(9):747-764. doi: 10.1016/s22150366(18)30095-6

Reis, T. L.; Dahl, C. M.; Barbosa, S. M.; Teixeira, M. R.; Delgado, P. G. G. (2016). Sobrecarga e participação de familiares no cuidado de usuários de Centros de Atenção Psicossocial. Saúde em Debate, 40(109), 70-85. doi: 10.1590/0103-1104201610906

Ryff, C. D. (1989). Hapiness is everything, or is it? Explorations on the meaning of psychological well-being. Journal of Personality and Social Psychology, 57(6), 1069-1081. doi: 10.1037/0022-3514.57.6.1069
Snyder, C. R.; Harris, C.; Anderson, J. R.; Holleran, S. A.; Irving, L. M.; Sigmon, S. T.; Yoshinobu, L. R.; Gibb, J.; Langelle, C.; \& Harney, P. (1991). The will and the ways: development of an individual differences measure of hope. Journal of Personality and Social Psychology, (60), 570-585. doi: 10.1037/0022-3514.60.4.570

Souza, C. M. de; Hidalgo, M. P. L. (2012). World Health Organization 5-item Well-Being Index: validation of the Brazilian Portuguese version. European archives of psychiatry and clinical neuroscience, 262(3), 239-244. doi: 10.1007/s00406-011-0255-x.2012.262(3):239-244

Tabeleão, V. P.; Tomasi, E.; Quevedo, L. (2014). Sobrecarga de familiares de pessoas com transtorno psíquico: níveis e fatores associados. Archives of Clinical Psychiatry, 41(3), 63-66. doi: 10.1590/0101-60830000000012

Trezise, A.; Mclaren, S.; Gomez, R.; Bice, B. \& Hodgetts, J. (2017). Resiliency among older adults: dispositional hope as a protective factor in the insomnia-depressive symptoms relation. Aging \& Mental Health, 22(8), 1088-1096. doi: 10.1080/13607863.2017.1334191

World Health Organization. (1998). WHOQOL user manual. Geneva: WHO. Disponível em: https://apps.who.int/iris/bitstream/handle/10665/77932/WHO_HIS_HSI_Rev.2012.03_eng. pdf? sequence $=1$ \&isAllowed $=y$

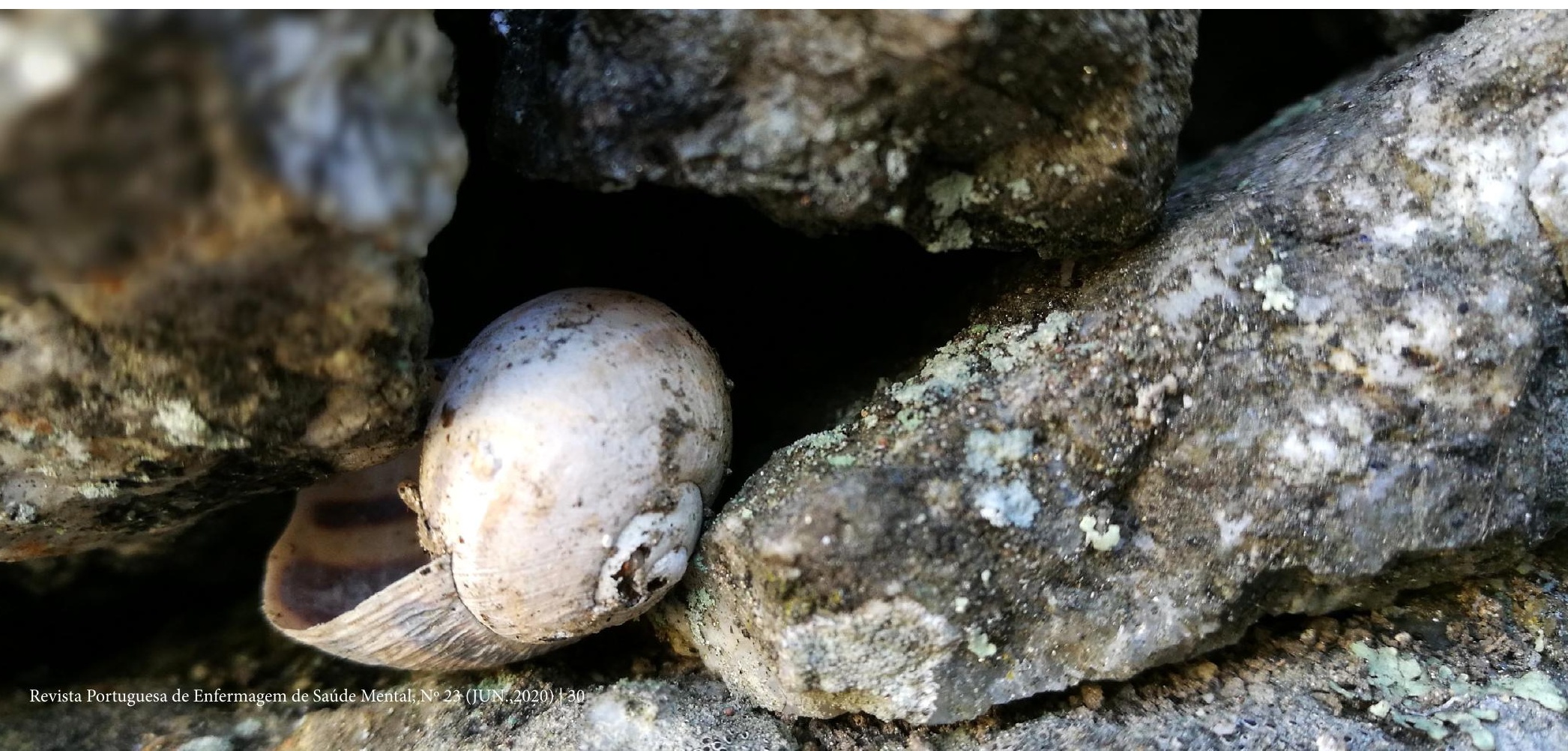

